(C) 2019 Universidad Nacional Autónoma de México, Facultad de Estudios Superiores Zaragoza.

Este es un artículo Open Access bajo la licencia CC BY-NC-ND (http://creativecommons.org/licenses/by-nc-nd/4.0/).

TIP Revista Especializada en Ciencias Químico-Biológicas, 22: 1-8, 2019.

DOI: 10.22201/fesz.23958723e.2019.0.195

\title{
Detección e identificación molecular de Pantoea vagans en flores de Dahlia sp.
}

\author{
Estrella Lara-Cortés ${ }^{1 *}$, Silvia Bautista- Baños ${ }^{2}$, \\ Laura Leticia Barrera-Necha ${ }^{2}$, Galdy Hernández-Zárate ${ }^{3}$ \\ y Renato León- Rodríguez ${ }^{4}$ \\ ${ }^{1}$ Laboratorio de Investigación del Complejo Regional Nororiental, Benemérita Universidad \\ Autónoma de Puebla. Calle Arias y Bulevard s/n. Teziutlán,73800, Puebla, México. 3702, ORCID: \\ 0000-0002-3581-0789. ${ }^{2}$ Instituto Politécnico Nacional-Centro de Desarrollo de Productos Bióticos, \\ Morelos, 62531, México, ORCID: 0000-0003-1556-0759, 0000-0002-5151-3004. ${ }^{3}$ Colegio de \\ Postgraduados-Campus Veracruz, Posgrado en Agroecosistemas, Mpio. de Manlio Fabio Altamirano, \\ 91960, Veracruz, México. ORCID: 0000-0002-0004-7410. ${ }^{4}$ Departamento de Medicina Genómica y \\ Toxicología Ambiental. Instituto de Investigaciones Biomédicas, UNAM, Coyoacán, 04510, Ciudad \\ de México, México, ORCID: 0000-0002-3679-3089. Emails: *laracort@hotmail.com
}

\begin{abstract}
RESUMEN
Las Dalias (Dahlia spp.) son flores nativas de Mesoamérica y endémicas de México. Su consumo como alimento es una práctica antigua, en la actualidad es escasa la información y reglamentación sanitaria para su comercialización y consumo. Al respecto, el objetivo del presente estudio fue identificar morfológica, bioquímica y molecularmente, bacterias de origen entérico asociadas con flores de Dalia. Los resultados de la caracterización morfológica revelaron la predominancia de bacilos cortos Gram negativos. A partir de la observación de la morfología colonial en medios de cultivo selectivos y diferenciales se identificaron como Escherichia coli y Salmonella spp. Sin embargo, el uso de un método comercial automatizado los clasifica como Enterobacter cancerogenus. Debido a que ambas pruebas arrojaron resultados distintos en cuanto a la identidad del microorganismo aislado, no fueron concluyentes. Por lo tanto, se recurrió a la caracterización molecular para la identificación del aislado bacteriano. La bacteria predominante en flores de Dalia, correspondió a Pantoea vagans (Número de acceso CP002206). Éste es el primer reporte de Pantoea vagans aislada de flores de Dalia.
\end{abstract}

Palabras Clave: Enterobacter cancerogenus, Dahlia, flores comestibles, Pantoea vagans.

\section{Detection and molecular identification of Pantoea vagans in flowers of Dahlia sp.}

\begin{abstract}
Dahlias (Dahlia spp.) are flowers native to Mesoamerica and endemic to Mexico. Its consumption as food is an ancient practice, at present there is little health information and regulation for its marketing and consumption. The objective of the study was to identify enteric bacteria associated morphologically, biochemically and molecularly with Dahlia flowers. The results of the morphological characterization revealed the predominance of short Gram-negative bacilli. From the observation of colonial morphology in selective and differential culture media Escherichia coli and Salmonella spp. were identified. However, the use of an automated commercial method classifieds them as Enterobacter cancerogenus. Due to the fact that the two tests used gave different results when identifying the isolated microorganism, the findings were inconclusive. Therefore molecular characterization was used to identify the bacterial isolate. Thus, the predominant bacterium in Dahlia flowers was Pantoea vagans (Access Number CP002206. 1). This is the first report of Pantoea vagans isolated from Dahlia flowers.
\end{abstract}

Key words: Enterobacter cancerogenus, Dahlia, edible flowers, Pantoea vagans.

Nota: Artículo recibido el 07 de agosto del 2019 y aceptado

el 05 de diciembre del 2019. 


\section{INTRODUCCIÓN}

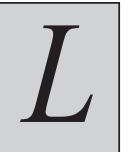

a flor de Dalia además de utilizarse como flor de ornato (nombrada la flor nacional mexicana), se ha usado como alimento en diversos platillos (Membre, Goubet \& Kubaczka,1995; Bye \& Linares, 2008; Díaz-López, 2017). Existen investigaciones que indican que esta flor posee algunos compuestos fenólicos de importancia para la salud humana (Bye \& Linares, 2008; Lara-Cortés et al., 2014). Por su parte, Anan'ina, Andreeva, Mycots \& Oganesyan (2009) señalan que la raíz de Dalia en forma deshidratada y pulverizada se ha empleado como parte de la gastronomía y la medicina herbolaria de México. Además, otros estudios indican que la inulina presente en sus raíces tuberosas sirve como fibra dietética y es considerada como un compuesto prebiótico que estimula especies bacterianas presentes en el colon como lactobacilos y bifidobacterias (Gibson \& Beatty, 1995; Gibson \& Roberfroid, 1995; López, Romero \& Ureta, 2001; Huebner, Wehlingy \& Hutkins, 2007; Puerta- García \& Mateos-Rodríguez, 2010).

Dentro de las características con las que debe contar un producto para ser utilizado como alimento, destaca su inocuidad. Esto es que no contenga objetos, sustancias o microorganismos que pongan en riesgo la salud de quién los consume. Al respecto, los productos vegetales frescos merecen atención especial, ya que la transmisión de enfermedades entéricas a través de su consumo es un problema ampliamente conocido (Berger et al., 2010; Santana-Legorreta, Villanueva-Carvajal, Morales-Rosales, Laguna-Cerda \& Domínguez-López, 2016; Alegbeleye, Singleton \& Sant'Ana, 2018). Cabe destacar que, en plantas, esta contaminación proviene principalmente del agua de riego, que funciona como vehículo de transmisión de agentes patógenos como bacterias, virus y protozoos procedentes de la materia fecal de origen humano y animal (Marchetti, Casadei \& Guerzoni, 1992; Leonila et al., 2017). Aunado a ello, la microbiota de los vegetales y frutas que crecen a ras del suelo está compuesta principalmente por microorganismos presentes de forma natural en el suelo, así como los que albergan las plantas en descomposición o bien por agentes microbianos que son incorporados después de la cosecha debido a la falta de higiene (Leonila et al., 2017).

Los principales agentes etiológicos relacionados con enfermedades transmitidas por vegetales y frutas frescas incluyen virus, bacterias, hongos y parásitos. Dentro de la población bacteriana que coloniza los vegetales frescoscortados, típicamente encontramos levaduras, hongos, bacterias ácido-lácticas y bacterias de los géneros Pseudomonas spp., Xanthomonas spp., Janthinobacterium spp., Enterobacter spp.; y actualmente, como consecuencia de diversas prácticas agrícolas también se ha aislado en este tipo de productos, Listeria monocytogenes y Aeromonas hydrophila (Miceli \& Settanni, 2019). Como principales enterobacterias (familia Enterobacteriaceae) que causan enfermedades transmitidas por el consumo de vegetales y frutas frescas, encontramos a $E$. coli, Enterobacter, Salmonella spp. y Shigella spp. (Alegbeleye et al., 2018).

Con respecto a la familia Enterobacteriaceae, ésta constituye un grupo grande y heterogéneo de bacterias Gram negativas, cuya reclasificación de varios de sus miembros ha sido propuesta actualmente con base a estudios de genómica comparativa (Alnajar \& Gupta, 2017). Las bacterias miembros de este grupo reciben su nombre por la localización habitual como comensales intestinales, aunque se trata de bacterias ubicuas, que se encuentran de forma universal en el suelo, el agua y la vegetación, así como formando parte de la microbiota intestinal normal de muchos animales, además del hombre (NOM093-SSA1-1994; Pachón-Cubillos, 2009). La importancia de este tipo de bacterias radica en que algunas son patógenos primarios para el ser humano (diversos patotipos diarréicos de E. coli, Salmonella spp., Shigella spp.) y en ocasiones cuando el paciente está inmunosuprimido (incluyendo pacientes alcohólicos y diabéticos), algunos miembros de esta familia se han reportado como causantes de infecciones, por lo que son considerados como patógenos oportunistas (E. coli, Enterobacter sp., Klebsiella sp., Citrobacter sp.), (Guentzel, 1996). Estas bacterias potencialmente patógenas, son comúnmente transmitidas por el agua contaminada, ocasionando diversas enfermedades de tipo gastrointestinal.

A nivel internacional, existe poca reglamentación con respecto al consumo de flores como alimento. En México, esta reglamentación es nula, no obstante, el Reglamento de control sanitario de productos y servicios de la Constitución, considera a las flores como un derivado de las frutas y hortalizas, por lo cual su objetivo es regular, controlar y fomentar la calidad sanitaria del proceso, importación y exportación, así como las actividades de servicios y establecimientos (DOF, 1999). La norma oficial mexicana NOM-093-SSA1-1994 (Mera-Ovando \& Boettler, 2006), establece los límites máximos permisibles para coliformes fecales presentes en ensaladas verdes crudas o en frutas que son preparados en establecimientos fijos.

Por lo anteriormente expuesto, el aseguramiento de la calidad microbiológica de flores comestibles de Dalia resulta de vital importancia en la obtención de un alimento que sea inocuo para el consumo humano. El objetivo de esta investigación fue aislar e identificar bacterias entéricas predominantes en flores de Dalia.

\section{MATERIALES Y MÉTODOS \\ Material vegetal}

Las flores de Dalia (Dahlia spp. var. Pompom) color púrpura fueron donadas por la Asociación Mexicana de la Dalia (AMD; Xochitla, Parque Ecológico, Tepotzotlán, México). Las flores fueron tratadas siguiendo los cuidados de riego, fertilización, control de plagas y enfermedades propios de la Dalia (JiménezMariña, 2015). 


\section{Medios de cultivo y aislamiento bacteriano}

Para el aislamiento bacteriano, fueron seleccionadas flores libres de daños mecánicos, insectos o signos de deterioro. Se pesaron $10 \mathrm{~g}$ de lígulas de flores de Dalias frescas y se incorporaron en $90 \mathrm{~mL}$ de caldo soya tripticaseína y caldo lactosado (MCD Lab.), respectivamente. Las muestras se homogenizaron y se incubaron a $35^{\circ} \mathrm{C}$ por 24 horas. Al término de la incubación, las muestras se sembraron por estría cruzada en cajas de Petri que contenían gelosa soya tripticaseína (MCD Lab). Las cajas se incubaron a $35^{\circ} \mathrm{C}$ por 24 horas. Pasado el tiempo de incubación, se realizó la tinción de Gram a los aislados obtenidos, así como la siembra de los aislados en medios de cultivo selectivos y diferenciales: gelosa de Xilosa, Lisina, Desoxicolato (XLD) (Becton, Dickinson de México), gelosa Verde Brillante (VB) (Laboratorios Britania), gelosa Mac Conkey (MCK) y gelosa Sulfito de Bismuto (SB) (MCD Lab.), con el propósito de observar las características morfológicas microscópicas y macroscópicas de las colonias aisladas. Cada medio de cultivo inoculado se incubó a $35^{\circ} \mathrm{C}$ durante 24 horas.

\section{Perfil bioquímico de los aislados bacterianos}

Las colonias aisladas de Dalia se caracterizaron bioquímicamente usando el sistema de identificación $B D B B L^{\mathrm{TM}}$ Crystal $^{\mathrm{TM}}$ Identification Systems Enteric/Nonfermenter ID Kit (Becton Dickinson). Se hicieron determinaciones adicionales como las pruebas de oxidasa e indol. Los resultados fueron registrados posterior a las 24 horas de incubación a $35^{\circ} \mathrm{C} \pm 2{ }^{\circ} \mathrm{C}$.

\section{Identificación molecular de los aislados bacterianos Preparación del cultivo}

La bacteria se cultivó aeróbicamente en gelosa soya tripticaseína (AST) a $35^{\circ} \mathrm{C} \pm 2{ }^{\circ} \mathrm{C}$ durante 18 -24 horas. Las células de un cultivo reciente (50-100 $\mathrm{mg}$ de biomasa bacteriana) (Toledo Hernández, 2013) se lavaron y resuspendieron en una solución de $\mathrm{Mg}_{2} \mathrm{SO}_{4}(10 \mathrm{mM})$. Y se mantuvieron hasta su utilización.

\section{Extracción y purificación del DNA de los aislados bacterianos} La extracción delDNA fue realizada usando el método comercial ZR Fungal/ Bacterial DNA ${ }^{\circledR}$ (Zymo Research), con base en las instrucciones del fabricante. La integridad delDNAcromosomal, y los productos de PCR se evaluaron por electroforesis en geles de agarosa $(1 \%)$ teñidos con bromuro de etidio $(0.5 \mu \mathrm{g} / \mathrm{mL})$ y se observaron en un transiluminador de luz ultravioleta a 254 nm. La concentración y pureza del DNA cromosómico se evaluó por espectrofotometría a 260/280 nm.

\section{Amplificación de los genes 16S $r R N A$ y gyrB del aislado bacteriano.}

Los genes $16 \mathrm{~S} r R N A$ y gyr $B$ se amplificaron por PCR, con el método comercial DreamTaq PCR Master Mix 2X (Thermo Fisher Scientific, México). Los oligonucleótidos se utilizaron a una concentración de 10 pmol cada uno y 100 ng del DNAmolde, en un volumen final de reacción fue de $50 \mu \mathrm{L}$. La secuencia de los oligos fueron: Pro K 63 5'-CAG GCC TAA CAC ATG
CAA GTC-3' y L1041 5' GCG TGT GTA CAA GAC CC 3' para el RNA 16 S (López et al., 2001), gyrB-UP1 5' GAA GTC ATC ATG ACC GTT CTG CAY GCN GGN AAR TTY GA 3' y gyrB-UP2r 5' AGC AGG GTA CGG ATG TGC GAG CCR TCN ACR TCN GCR TCN GTC AT 3', para amplificar gyrB ( Weinthal et al., 2007), gyrB-UP1 Sec 5' GAA GTC ATC ATG ACC GTT CTG CA 3' y gyrB-UP2r Sec 5' AGC AGG GTA CGGATG TGC GAG CC 3', para secuenciar gyrB (Weinthal et al, 2007).La programación del termociclador (Lab. Net, Mod. Axigen MaxigeneII) consistió de una desnaturalización inicial a $95{ }^{\circ} \mathrm{C}$ por $5 \mathrm{~min}$, seguida por 30 ciclos de $95^{\circ} \mathrm{C}$ por $1 \mathrm{~min}$, desnaturalización; $54^{\circ} \mathrm{C}(16 \mathrm{~S} r R N A)$ y $58^{\circ} \mathrm{C}($ gyr $B)$ por $40 \mathrm{seg}$, alineamiento; y extensión a $72^{\circ} \mathrm{C}$ por $80 \mathrm{seg}$; y al final un ciclo de extensión por $10 \mathrm{~min}$ a $72^{\circ} \mathrm{C}$. Los productos amplificados de los genes fueron: $\sim 1.3 \mathrm{pb}$ para $16 \mathrm{~S} r R N A \mathrm{y} \sim 1.2 \mathrm{pb}$ para $g y r \mathrm{~B}$. Los productos de PCR fueron purificados a partir de un gel de agarosa de bajo punto de fusión (1\%) con un método comercial

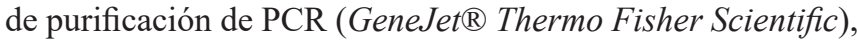
con base en las instrucciones del fabricante. Los productos de PCR fueron secuenciados (en ambas direcciones) en la unidad de síntesis y secuenciación del DNA del Instituto de Biotecnología, UNAM. Las secuencias se analizaron y editaron con el software ApE. Aplasmid Editor v2.0.45. Posteriormente, las secuencias fueron alineadas y comparadas para determinar la similitud con otras secuencias disponibles en la base de datos del Gen Bank (http://www.nlm.nih.gov) a través del Centro Nacional para la Información Biotecnológica (NCBI) de EE.UU., utilizando el programa bioinformático BLAST (http:www.ncbi.nlm.nih. gov/BLAST).

\section{Construcción del árbol filogenético}

Para la construcción del árbol filogenético se utilizaron las secuencias obtenidas de la base de datos del Gen Bank, correspondientes a los genes del marcador molecular gyr $B$, que codifican para la enzima DNA girasa. Elárbol se enraizó tomando como referencia el clado externo Azotobacter vinelandii. Elárbol filogenético fue construido con base en los valores de similitud de las secuencias, utilizando el método Neighbor Join (JN) y para el cálculo de las distancias genéticas se empleó el método Jukes-Cantor, ambos del programa de acceso libre MEGA 7 (http://www.megasoftware.net).

\section{RESULTADOS Y DISCUSIÓN}

\section{Obtención de aislados bacterianos}

En los resultados de todas las muestras analizadas se observaron únicamente bacilos Gram negativos, delgados y cortos (Figura 1), al aislado se le denominó ET1. Las características del aislado ET1 de flores de Dalia se muestran en la Figura 2 y Tabla I. Los resultados de las características de la morfología colonial del aislado ET1 en medios de cultivo selectivos y diferenciales descartan que el microorganismo aislado, sea E. coli o Salmonella spp. (Balows, 1991). Esto debido a que no presenta las características típicas de estos microorganismos en los medios de cultivo utilizados (Tabla I). 


\section{Perfil bioquímico del aislado bacteriano}

Los resultados de las pruebas bioquímicas aplicadas al aislado bacteriano ET1 de flores de Dalia se muestran en la Tabla II, los cuales sugieren que corresponden a Enterobacter cancerogenus, bacteria que forma parte de la familia de las enterobacterias, recientemente propuesto por Adeolu, Alnajar, Naushad \& Gupta (2016). Esta bacteria es considerada como un patógeno oportunista hospitalario, resistente a álcalis y antibióticos (Springer, Stockman, Teschner, Roberts \& Bottger, 1996). Debido a que los resultados morfológicos y bioquímicos no coinciden con el perfil bioquímico y por tanto no pueden ser concluyentes, se procedió a la identificación del aislado ET1 de flores de Dalia con marcadores moleculares.

\section{Identificación molecular de los aislados bacterianos ET1}

El resultado del alineamiento de la secuencia $16 \mathrm{~S} r R N A$ del aislado bacteriano ET1 de flores de Dalia, mostró un $88 \%$ de identidad con diferentes especies del género Pantoea. Este hecho ha sido previamente señalado, indicando que las interrelaciones de los miembros del orden Enterobacteriales, se describen de forma limitada con árboles filogenéticos basados en este gen (Adeolu et al., 2016). Sin embargo, para el alineamiento del gen $g y r B$, se demostró una identidad de un $98 \%$ con cepas de Pantoea vagans, la cual incluye la cepa tipo $P$. vagans $C 9-1$, de la cual se tiene su genoma secuenciado (Número de acceso CP002206). Estos resultados sugieren que el aislado ET1 aislado de flores de Dalia corresponde a Pantoea vagans ET1. Se construyó un árbol filogenético (Figura 3), con la secuencia de gyrB de P. vagans ET1, y de otras enterobacterias que se obtuvieron del Gen Bank(NCBI). En el árbol se observa que $P$. vagans ET1 aislada de flores de Dalia, se agrupa con otras cepas de $P$. vagans, la cual incluye a $P$. vagans PV989 (CP028349), cepa con la que exhibió la mayor similitud en los resultados de alineamiento con gyrB. En la identificación de aislados bacterianos con marcadores moleculares, el más usado es el $16 \mathrm{~S} r R N A$, pero como ya se mencionó, hay grupos bacterianos donde la identificación está limitada (Adeolu et al., 2016). En cambio gyrB presenta una mayor resolución tanto en grupos de Bacillus (Weinthal et al., 2007) como en este estudio. También comparando la identificación morfológica y bioquímica (fenotipo) con la identificación a nivel molecular (genotipo) de $P$. vagans ET1, se observa que éstas no coinciden. Al respecto Springer y colaboradores (1996) mencionan que el fenotipo (características morfológicas y bioquímicas) de una especie bacteriana, no es una propiedad absoluta, si no que puede exhibir una gran variabilidad. Esta variabilidad depende en gran parte de las condiciones ambientales así, como del hospedero del cual se aísle el microorganismo. De tal modo que las bacterias se caracterizan por mostrar una gran plasticidad fenotípica y metabólica. Además, la presencia de plásmidos (moléculas del DNA distintas al cromosoma bacteriano), aporta información genética adicional, aunque no esencial para la vida de diversas bacterias que los albergan. Los plásmidos, les confieren nuevas propiedades fenotípicas y en algunos casos,

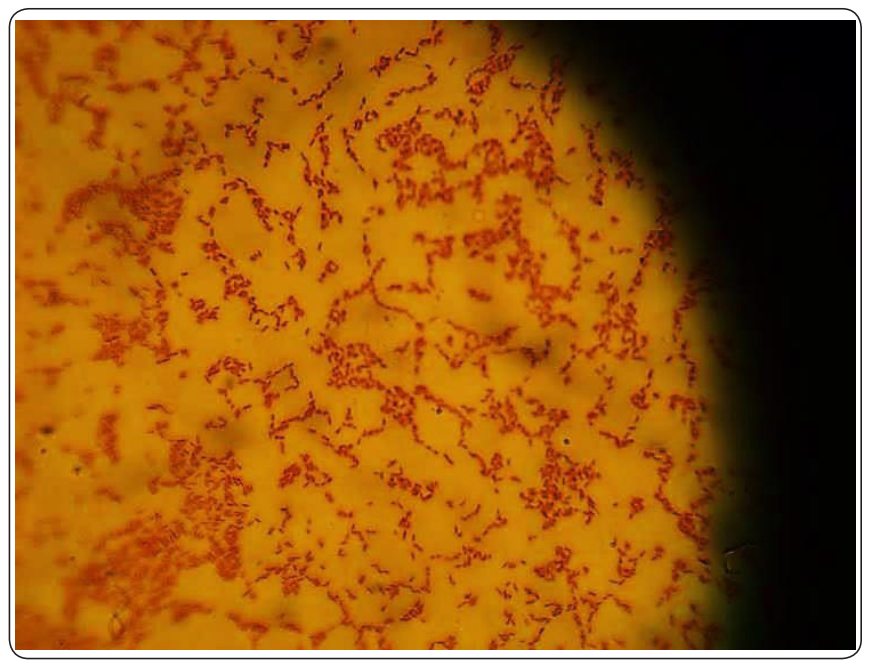

Figura 1. Bacilos Gram negativos del aislado ET1 (Pantoea vagans) de flores de Dalia. Aumento $100 \mathrm{X}$.

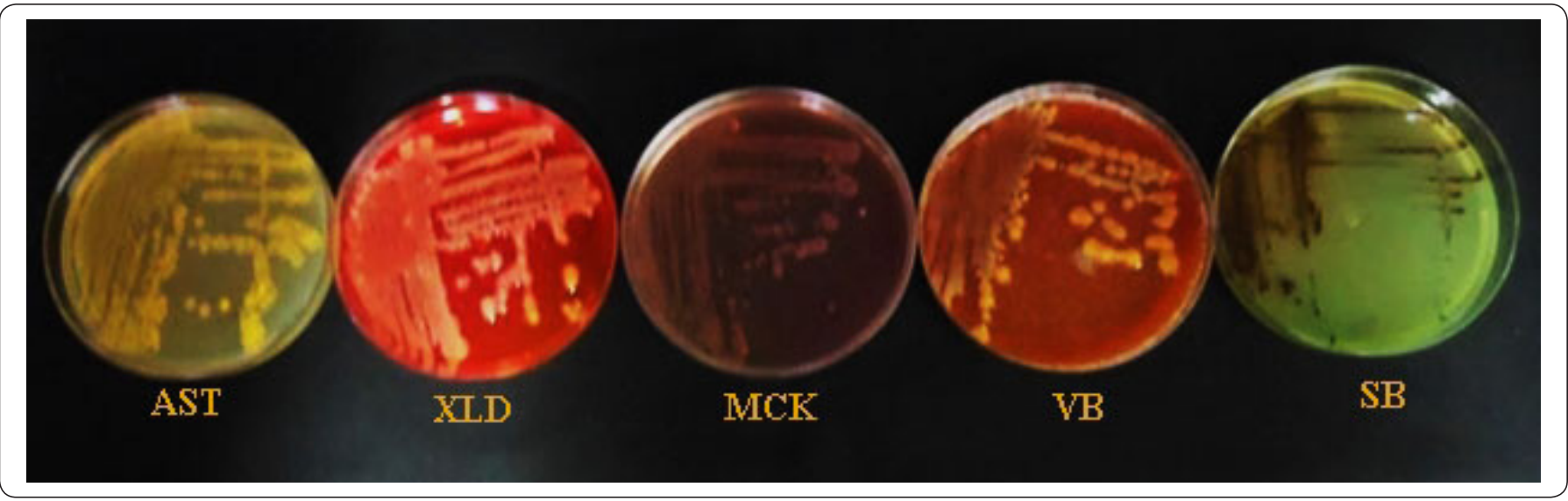

Figura 2. Crecimiento del aislado ET1 (Pantoea vagans) en diferentes medios de cultivo. Gelosa soya tripticaseína (AST), agar XLD, gelosa Mac Conkey (MCK), gelosa Verde Brillante (VB) y gelosa Sulfito de Bismuto (SB). 


\begin{tabular}{|c|c|c|c|}
\hline \multirow[b]{2}{*}{ Medio de cultivo } & \multicolumn{3}{|c|}{ AISLADOS BACTERIANOS DE Dahlia sp. } \\
\hline & Aislado ET1 & E. coli $^{*}$ & Salmonella spp. * \\
\hline Soya Tripticaseína & $\begin{array}{l}\text { Colonias amarillas, circulares, } \\
\text { rugosas, convexas, mucoides y } \\
\text { húmedas. }\end{array}$ & - & - \\
\hline $\begin{array}{l}\text { Xilosa-Lisina- } \\
\text { Desoxicolato (XLD) }\end{array}$ & $\begin{array}{l}\text { Colonias circulares, amarillas, } \\
\text { con halo transparente, convexas, } \\
\text { mucoides, superficie lisa, } \\
\text { brillante y húmeda. } \\
\end{array}$ & $\begin{array}{l}\text { Colonias grandes, amarillas } \\
\text { con o sin precipitado de } \\
\text { bilis. }\end{array}$ & $\begin{array}{l}\text { Colonias de color rojo con el centro } \\
\text { negro debido a la producción de } \mathrm{H}_{2} \mathrm{~S} \text {. }\end{array}$ \\
\hline Mac Conkey & $\begin{array}{l}\text { Colonias rosas brillantes, } \\
\text { convexas con bordes irregulares, } \\
\text { superficie lisa y consistencia } \\
\text { mucoide. }\end{array}$ & $\begin{array}{l}\text { Colonias de color rosa a } \\
\text { rojo con precipitado de } \\
\text { sales biliares que rodea a } \\
\text { las colonias }\end{array}$ & Colonias incoloras \\
\hline Verde Brillante & $\begin{array}{l}\text { Colonias grandes, circulares, } \\
\text { convexas, amarillas con } \\
\text { un pequeño halo amarillo } \\
\text { consistencia mucoide, superficie } \\
\text { lisa y brillante. Fermentadoras } \\
\text { de la lactosa. } \\
\end{array}$ & $\begin{array}{l}\text { Amarillo-verdosas sobre } \\
\text { fondo amarillento } \\
\text { Crecimiento entre nulo y } \\
\text { moderado, colonias entre } \\
\text { amarillas y amarillas } \\
\text { verdosas, halo amarillo } \\
\end{array}$ & $\begin{array}{l}\text { Colonias pequeñas, incoloras, } \\
\text { rosadas o fucsias, transparentes u } \\
\text { opacas, sobre el medio coloreado de } \\
\text { rosado a rojo }\end{array}$ \\
\hline Sulfito de Bismuto & $\begin{array}{l}\text { Colonias negras circulares } \\
\text { convexas con brillo metálico y } \\
\text { superficie rugosa }\end{array}$ & $\begin{array}{l}\text { Crecimiento inhibido o } \\
\text { colonias verdes }\end{array}$ & $\begin{array}{l}\text { Colonias marrones o negras, algunas } \\
\text { veces con brillo metálico alrededor } \\
\text { de las colonias. Algunas cepas } \\
\text { producen colonias verdes con poco o } \\
\text { ningún oscurecimiento. }\end{array}$ \\
\hline
\end{tabular}

*Manual of Clinical Microbiology. (1991). 5a Edición, American Society for Microbiology, Washington, D. C.

Tabla I. Características morfológicas en medios de cultivo selectivos y diferenciales del aislado ET1 de flores de Dalia y características típicas de los microorganismos en los medios de cultivo utilizados.

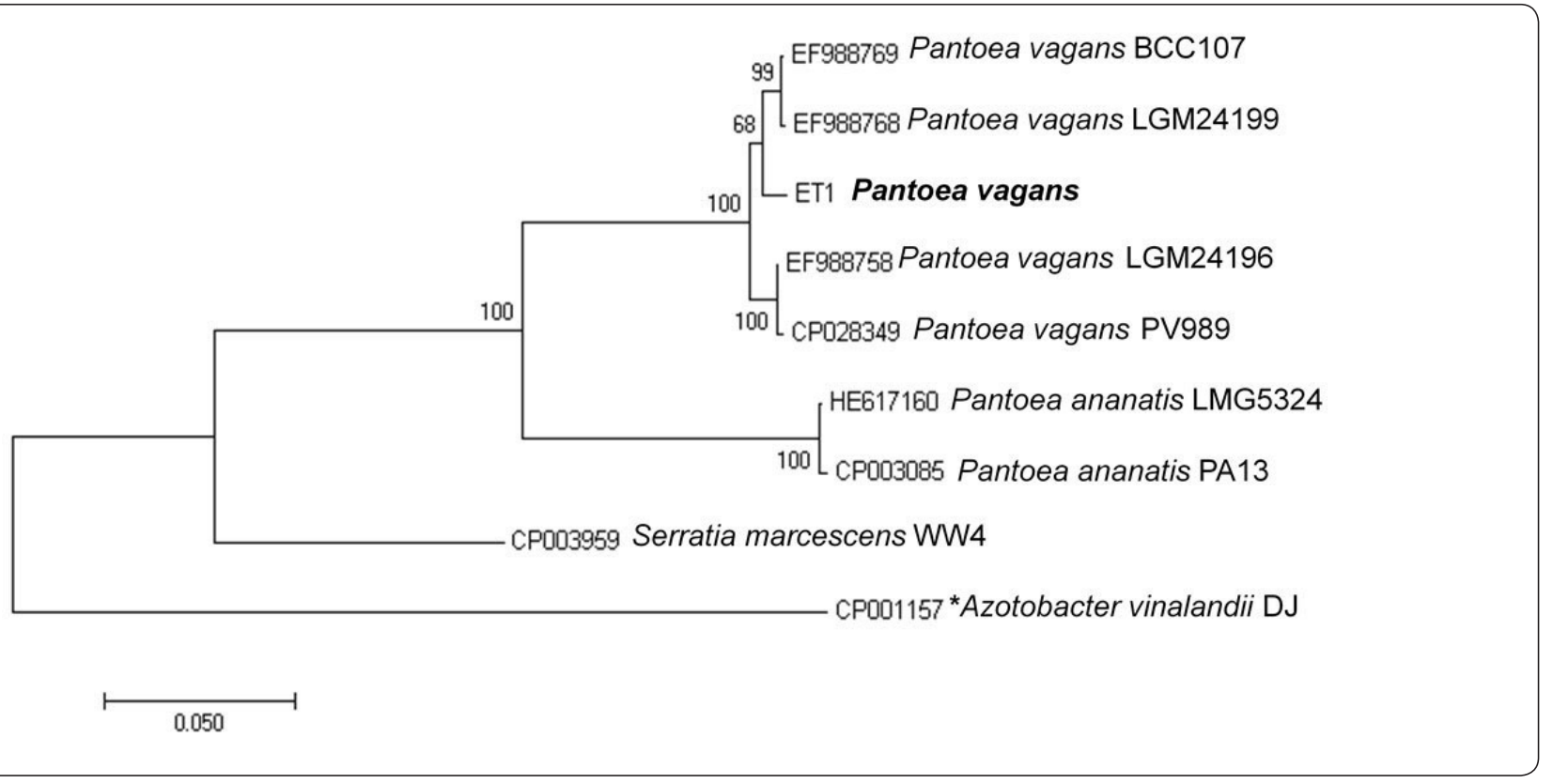

Figura 3. Árbol filogenético utilizando la secuencia del gen DNA girasa ( $g y r B)$ de secuencias bacterianas obtenidas de la base de datos Gen Bank y de la bacteria aislada (P. vagans, ET1) de flores de Dahlia sp. La bacteria Azotobacter vinalandii DJ (*) se incluyó como clado externo. El árbol fue construido usando el método Neighbor-Join. El número en los nodos indica el valor del porcentaje bootstrap basado en 1,000 réplicas. La escala bajo el árbol filogenético indica el número de sustituciones por posición de los nucleótidos. 
son útiles a las bacterias para su adaptación al crecimiento en determinados ambientes. Éstos pueden o no estar presentes en la célula bacteriana y por tanto son en parte responsables de la inestabilidad fenotípica. Tomando en cuenta la amplia diversidad fenotípica y genotípica que presentan las bacterias, es necesario realizar la identificación no sólo cultural y bioquímica, sino que también se debe recurrir a métodos moleculares (basados en la secuenciación genética) que hagan más robusta la identificación de este tipo de microorganismos.

Por otro lado, respecto al microorganismo aislado e identificado en este trabajo, $P$. vagans, pertenece a la Clase de las Proteobacterias Gamma $(\gamma)$. Anteriormente, se consideraba dentro de la familia Enterobacteriaceae. Sin embargo,

\begin{tabular}{|c|c|c|c|}
\hline \multirow[b]{2}{*}{ Prueba bioquímica } & \multicolumn{3}{|c|}{ RESULTADOS } \\
\hline & Aislado ET1 & E. coli $^{*}$ & Salmonella spp. * \\
\hline Arabinosa & Positivo & Variable & Positivo \\
\hline Manosa & Positivo & Positivo & Positivo \\
\hline Sacarosa & Negativo & Negativo & Negativo \\
\hline Melibiosa & Positivo & Positivo & Positivo \\
\hline Ramnosa & Positivo & Positivo & Positivo \\
\hline Sorbitol & Negativo & Positivo & Positivo \\
\hline Manitol & Positivo & Positivo & Positivo \\
\hline Adonitol & Negativo & Negativo & Negativo \\
\hline Galactosa & Positivo & Positivo & Positivo \\
\hline Inositol & Negativo & Negativo & Negativo \\
\hline p-n-p-fosfato & Positivo & Variable & Negativo \\
\hline p-n-p $\alpha$ - $\beta$-glucósido & Positivo & Negativo & Negativo \\
\hline p-n-p- $\beta$-galactósido & Positivo & Positivo & Negativo \\
\hline Prolinenitroanilide & Negativo & Negativo & Negativo \\
\hline p-n-p bis-fosfato & Positivo & Variable & Positivo \\
\hline Prueba Bioquímica & $\begin{array}{c}\text { Aislados de Flores de } \\
\text { Dalia }\end{array}$ & E. coli* & Salmonella spp.* \\
\hline p-n-p-xilósido & Negativo & Negativo & Negativo \\
\hline p-n-p- $\alpha$-arabinósido & Positivo & (Negativo) $^{\mathrm{p}}$ & Negativo \\
\hline p-n-p-fosforilcolina & Negativo & Negativo & Negativo \\
\hline p-n-p- $\beta$-glucurónido & Negativo & Positivo & Positivo \\
\hline p-n-p-N-acetil glucosaminida & Positivo & Negativo & Negativo \\
\hline$\gamma$ - $L$-glutamil p-nitroanilida & Positivo & Negativo & Positivo \\
\hline Esculina & Positivo & Negativo & Negativo \\
\hline p-nitro-DL-fenilalanina & Positivo & Negativo & Negativo \\
\hline Urea & Negativo & Negativo & Negativo \\
\hline Glicina & Negativo & Negativo & Negativo \\
\hline Citrato & Positivo & Negativo & Positivo \\
\hline Ácido Malónico & Positivo & Negativo & Negativo \\
\hline Cloruro trifeniltetrazolium & Positivo & ${\text { (Positivo })^{\mathrm{p}}}$ & Positivo \\
\hline Arginina & Positivo & Variable & Positivo \\
\hline Lisina & Positivo & Positivo & Positivo \\
\hline
\end{tabular}

*Manual of Clinical Microbiology. (1991). 5a Edición, American Society for Microbiology, Washington, D. C. presultado más común.

Tabla II. Pruebas bioquímicas para la identificación del aislado ET1 de Dahlia. 
recientemente, ha sido propuesta como un grupo separado del clado Klebsiella, en el clado Erwinia-Pantoea y dentro de un orden nuevo denominado Enterobacterales (Adeolu etal.,2016; Alnajar \& Gupta, 2017).

P. vagans, es aislada de diversas áreas geográficas y fuentes ecológicas como plantas, semillas, agua, humanos y suelo (Koneman, Allen, Janda, Schreckenberger \& Winn, 1997). Pantoea vagans (syn. Pantoea agglomerans, Erwinia herbicola) (Leonila et al., 2017) es una bacteria epífita común en plantas. La cepa C9-1, aislada de manzana Malus domestica 'Jonathan' (Michigan) (Ishimaru, Klos \& Brubaker, 1988) está registrada como Blight Ban C9-1 (Nufarms America Inc., Burr Ridge, $I L)$, para biocontrol del fuego bacteriano causado por la enterobacteria Erwinia amylovora. Sin embargo, a pesar de no ser una bacteria patógena como tal, algunos aislados han sido señalados como patógenos tumorogénicos (Grimont \& Grimont, 2005; Weinthal et al., 2007). Como un patógeno oportunista en humanos, $P$. agglomerans puede ocurrir esporádicamente o en brotes. A principios de los años 1970, fue implicada en un brote de septicemia significativo en EE. UU. y Canadá, causado por cierres contaminados en las botellas de líquidos de infusión, donde 25 hospitales estuvieron involucrados, con 378 casos señalados (Maki et al., 1976). Koo y colaboradores (2006), en un estudio señalaron un total de 22 aislados de cepas de Pantoea, como agentes inusuales y causantes de infecciones clínicas. Las cepas fueron aisladas de cultivos sanguíneos de 9 pacientes, y un aislamiento del oído de un paciente, en el plazo de un mes en un hospital de tercer nivel. Considerando esta información, este hallazgo es de suma importancia debido a que las flores de Dalia se pueden consumir. Se sugiere monitorear la presencia de este microorganismo y eliminarlo, ya que puede representar un factor de riesgo para la salud humana.

\section{Conclusiones}

Con base en los resultados obtenidos el microorganismo aislado de flores de Dalia fue Pantoea vagans (Número de acceso CP002206). Con la consulta a la literatura realizada, este es el primer informe del aislamiento de $P$. vagans de flores de Dalia con fines de florifagia. Es importante que, en la floricultura, los productores que las cultivan con fines alimenticios sigan las buenas prácticas de higiene para garantizar la inocuidad microbiológica de sus productos. Debido a que la flor de Dalia está destinada al consumo como alimento, es necesario buscar alternativas de compuestos antimicrobianos contra $P$. vagans para que éstas sean inocuas para el consumidor o bien no causen problemas de sanidad vegetal en la flor.

\section{CONFLICTO DE INTERESES}

Los autores declaran que no existen conflictos de intereses.

\section{FinanCiamiento}

Este trabajo fue financiado por el Consejo Nacional de Ciencia y Tecnología (CONACYT) número de registro de 46565 y el
Instituto Politécnico Nacional (IPN) por medio del Programa Institucional de Formación de Investigadores de la Secretaría de Investigación y Posgrado (SIP) número de proyecto SIP: 20131176.

\section{REFERENCIAS}

Adeolu, M., Alnajar, S., Naushad, S. \& Gupta, S. (2016). Genomebased phylogeny and taxonomy of the 'Enterobacteriales': proposal for Enterobacterales ord. nov. divided into the families Enterobacteriacea, Erwiniacea fam. Nov., Pectobacteriaceae fam. Nov., Yersiniaceae fam. Nov., Hafniaceae fam. Nov., Morganellaceae fam. Nov., and Budviciaceae fam. nov.International Journal of Systematic and Evolutionary Microbiology, 66, 5575-5599. https:// dx.doi.org/10.1099/ijsem.0.001485.

Alegbeleye, O. O., Singleton, I. \& Sant' Ana, A. S. (2018). Sources and contamination routes of microbial pathogens to fresh produce during field cultivation: A review. Food Microbiology, 73, 177-208. https://doi.org/10.1016/j. fm.2018.01.003.

Alnajar, S. \& Gupta, R. S. (2017). Phylogenomics and comparative genomic studies delineate six main clades within the family Enterobacteriaceae and support the reclassification of several polyphyletic members of the family. Infection Genetics and Evolution, 54, 108 -127. https://doi.org/10.1016/j. meegid.2017.06.024.

Anan'ina, N. A, Andreeva, O. A, Mycots, L. P. \& Oganesyan, E. T. (2009). Standardization of inulin extracted from Dahlia single tubers and some physicochemical properties of inulin. Pharmacology Chemistry Journal, 43, 157-159. https://doi. org/10.1007/s11094-009-0261-8.

Balows,A.(1991). Manual of Clinical Microbiology. Washington, D.C.: American Society for Microbiology.

Berger, C. N., Sodha, S. V., Shaw, R. K., Griffin, P. M., Pink, D., Hand, P. \& Frankel, G. (2010). Fresh fruit and vegetables as vehicles for the transmission of human pathogens. Environmental Microbiology, 12, 2385-2397. DOI: 10.1111/j.1462-2920.2010.02297.x.

Bye, R. \& Linares, E. (2008). La dalia, flor nacional de México. CONABIO. Biodiversitas, 76, 13-15.

Diario Oficial de la Federación de México (DOF), (1999). Reglamento de Control Sanitario de Productos y Servicios. Secretaría de salud. Segunda Edición; México, D. F. http://www.salud.gob.mx/unidades/cdi/nom/compi/ resps.htmlS.

Díaz-López, K. A. (2017). Usos gastronómicos de la planta Dalia (Dahlia spp.). Tesis de Licenciatura en Gastronomía. Universidad de Ciencias y Artes de Chiapas. Tuxtla Gutiérrez, Chiapas, México. (pp. 1-97).

Gibson, G. R. \& Beatty, E. R. (1995). Cummings, J. Selective fermentation of bifidobacteria in the human colon by oligofructose and inulin. Gastroenterology, 108, 975-982.

Gibson, G. R. \& Roberfroid, M. B. (1995). Dietary modulation of the human colonic microbiota: Introducing the concept 
of prebiotics. Journal of Nutrition, 125, 1401-1412. https:// doi.org/10.1093/jn/125.6.1401.

Grimont, P.A.D. \& Grimont, F. (2005). Genus Pantoea. En: Brenner D. J., Krieg, N. R., Staley J. T. (Ed.). Bergey's Manual of Systematic Bacteriology. The Proteobacteria. (pp. 713-720) Nueva York: Springer- Verlag.

Guentzel, M. N. (1996). Escherichia, Klebsiella, Enterobacter, Serratia, Citrobacter, and Proteus. En: Baron, S. (Ed). Medical Microbiology. EE. UU: Churchill Livingstone.

Huebner, J., Wehlingy, R. L. \& Hutkins, R.W. (2007). Functional activity of commercial prebiotics. International Dairy Journal, 17, 770-775. https://doi.org/10.1016/j. idairyj.2006.10.006.

Ishimaru, C. A., Klos, E. J. \& Brubaker, R. R. (1988). Multiple antibiotic production by Erwinia herbicola. Phytopathology, 78, 746-750. https://www.apsnet.org/publications/ phytopathology/backissues/Documents/1988Articles/ Phyto78n06_746.PDF.

Jiménez-Mariña, L. (2015). El cultivo de la Dalia. Cultivos Tropicales, 36, 107-115. http://www.redalyc.org/ pdf/1932/193237111014.pdf.

Koneman, E. W., Allen, S. D., Janda, W. M., Schreckenberger, P. C. \& Winn, W.C. (1997). The Enterobacteriaceae. En: Koneman, E. W., Allen, S. D., Janda, W. M., Schreckenberger, P. C., Winn, W. C. (Ed.). Color atlas and textbook of diagnostic microbiology. (171-252) Filadelfia: Lippincott-Raven.

Koo, H.-S., Kim, J.-S., Eom, J.-S., You, J.-Y., Park, J.-Y., Kim, H-S., Song, W., Cho, H.C. \& Lee, K. M. (2006). Pseudo outbreak of Pantoea species bacteremia associated with contaminated cotton pledgets. American Journal of Infection Control, 34, 443-446. https://doi.org/10.1016/j.ajic.2006.07.001.

Lara-Cortés, E., Martín-Belloso, O., Osorio-Díaz, P., BarreraNecha, L. L., Sánchez-López, J. A. \& Bautista-Baños, S. (2014). Actividad antioxidante, composición nutrimental y funcional de flores comestibles de Dalia. Revista Chapingo Serie Horticultura, 20, 101-116.

Leonila, M. L. A., Vilar, J. C., Barbosa da Silveira, A., Gomes de Almeida, F. C., de Lima e Silva, T. A. \& de Campos-Takaki, G. M. (2017). Isolation, Identification, Characterization and Enzymatic Profile of the New Strain of Pantoea agglomerans. International Journal of Current Microbiology and Applied Sciences, 6, 4152-4163.https://doi.org/10.20546/ ijcmas.2017.611.487.

López, V. L, Romero, R. J. \& Ureta, V.F. (2001). Tratamientos de desinfección de lechugas (Lactuca sativa) y frutillas (Fragaria chiloensis). Archivos Latinoamericanos de Nutrición, 51, 376-381.

Marchetti, R., Casadei, M.A. \& Guerzoni, M. E. (1992). Microbial population dynamics in ready-to-use vegetables salads. Italian Journal of Food Science, 2, 97-108.

Maki, D. G., Rhame. F. S., Mackel, D.C. \& Bennett, J. V. (1976). Nationwide epidemic of septicemia caused by contaminated intravenous products. Epidemiologic and clinical features. The American Journal of Medicine, 60, 471-485.
Membre, J. M., Goubet, D. \& Kubaczka, M. (1995). Influence of salad constituents on growth of Pseudomonas marginalis: a predictive microbiology approach. Journal of Applied Bacteriology, 79, 603-608. https://doi. org/10.1111/j.1365-2672.1995.tb00943.x.

Mera Ovando, L. M. \& Boettler, R. B. (2006). La Dahlia una belleza originaria de México. Revista Digital Universitaria, 7,1-11. [On-line] http://www.revista.unam.mx/vol.7/num11/ art90/int90.htm.

Miceli, A. \& Settanni, L. (2019). Influence of agronomic practices and pre-harvest conditions on the attachment and development of Listeria monocytogenes in vegetables. Annals of Microbiology, 69, 185-199.

Norma Oficial Mexicana NOM-093-SSA1-1994. Bienes y servicios. Prácticas de higiene y sanidad en la preparación de alimentos que se ofrecen en establecimientos fijos. 1994; México, D. F. http://www.salud.gob.mx/unidades/cdi/ nom/093ssa14.html.

Pachón-Cubillos, D. A. (2009). Aislamiento, identificación y serotipificación de Enterobacterias del género Salmonella en una población de Crocodylus intermedius y Testudinos mantenidos en cautiverio en la estación de Biología Tropical Roberto Franco E.B.T.R.B. de la Facultad de CienciasUniversidad Nacional de Colombia en Villavicencio-Meta. Tesis de Microbiólogo Agrícola y Veterinario. Pontificia Universidad Javeriana. Facultad de Ciencias Básicas. Bogotá, D.C. (pp. 1-115). https://javeriana.edu.co/biblos/ tesis/ciencias/tesis198.pdf.

Puerta-García,A. \& Mateos-Rodríguez, F. (2010). Enterobacterias. Medicine, 10, 3426-3431. http://www.facmed.unam.mx/ deptos/microbiologia/pdf/Enterobacterias_Medicine2010. pdf.

Santana Legorreta, S., Villanueva-Carvajal,A., Morales-Rosales, E. J., Laguna-Cerda, A. \& Domínguez-López, A. (2016). Extracción y evaluación de inulina a partir de dalias silvestres mexicanas (Dahlia coccinea Cav.). Revista Internacional de Botánica Experimental, 85, 63-70. [On-line].

Springer, B., Stockman, L., Teschner, K., Roberts, G. D. \& Bottger, E. C. (1996). Two laboratory collaborative study on identification of mycobacteria: molecular versus phenotypic methods. Journal of Clinical Microbiology, 34, 296-303. [On-line] https://www.ncbi.nlm.nih.gov/pmc/articles/ PMC228786/pdf/340296.pdf.

Toledo Hernández, E. (2013). Identificación, caracterización y evaluación de Rizobacterias para el control de hongos fitopatógenos de semillas de Jatropha curcas L. Tesis de maestría. Centro de Desarrollo de Productos Bióticos-Instituto Politécnico Nacional, Yautepec, Morelos, México. (pp. 1-106)

Weinthal, D. M., Barash, I., Panijel, M., Valinsky, L., Gaba, V. \& Manulis- Sasson, S. (2007). Distribution and replication of the pathogenicity plasmid PPATH in diverse populations of the gall-forming bacterium Pantoea agglomerans. Applied and Environmental Microbiology, 73, 7552-7561. DOI: 10.1128/AEM.01511-07. 